\title{
INFLAMMATORY MYOFIBROBLASTIC TUMOR OF SIGMOID COLON- A CASE REPORT
}

Samir. S. Deolekar, Harsh R. Sheth, Shrikant Perka, Kushal Bairoliya
1. Associate Professor. Department of General Surgery, KEM Hospital \& Research Centre.
2. Senior Resident. Department of General Surgery, KEM Hospital \& Research Centre.
3. Senior Resident. Department of General Surgery, KEM Hospital \& Research Centre.
4. Junior Resident. Department of General Surgery, KEM Hospital \& Research Centre.

\section{CORRESPONDING AUTHOR:}

Dr. Harsh R. Sheth, 39, Napean sea road, Matru Ashish, $7^{\text {th }}$ floor, A-2, Mumbai-400026.

E-mail: harsh.sheth@me.com

Ph: 00919819388836.

ABSTRACT: Inflammatory myofibroblastic tumor (IMT) comprise of rare group of lesions characterized histologically by acute and chronic inflammatory cells with variable degree of fibrous stroma. Also called as Plasma cell granuloma or Inflammatory pseudotumor IMTs are most often seen in lung of young adults(2). The extrapulmonary sites are rare, and are found mostly in liver, spleen and small intestinal mesentery. Large bowel mesentery, rectum and appendenceal sites of IMTs are not often known ${ }^{(1,3,4)}$.We present a case of IMT of sigmoid mesocolon in 27 years old young female patient whose symptoms improved after surgical resection of tumor.

KEY WORDS: Inflammatory myofibroblastic tumour, Sigmoid colon

CASE REPORT: We report a case of 27 years old female patient who presented to us with 2 months history of intermittent, high grade fever not responding to medical treatment, malaise, weight loss and loss of appetite. She also complained of dull aching pain in lower abdomen since 1 month which responded to regular doses of analgesics. There was no significant bowel or bladder complaints. Patient was known case of hypothyroidism diagnosed since 5 years and was on T. Eltroxin.

On general examination she was pale, and initial vital signs showed temperature of $40^{\circ} \mathrm{C}$, pulse of $92 / \mathrm{min}$, blood pressure of $130 / 80 \mathrm{~mm}$ of $\mathrm{Hg}$ and respiratory rate of $14 / \mathrm{min}$. On Per abdomen examination there was an ill defined, nontender, nonpulsatile, and fixed lump in the hypogastrium measuring approximately $10 \times 10 \mathrm{~cm}$ in size .

Her initial laboratory investigations showed haemoglobin of 7gm\%,Leucocyte count of $13,000 / \mathrm{mm}^{3}$, and raised ESR. Her platelet counts, liver function and renal function tests were within normal limits .

USG on admission showed $9 \times 9 \times 8 \mathrm{~cm}$ mass arising in the pelvis in close proximity to sigmoid colon. There was no ascites or significant abdominal lymphadenopathy. Liver was grossly normal.

Computed tomography showed similar size, homogenously enhancing ,exophytic mass arising from the sigmoid colon probably due to obliteration of fat planes between colon and the mass. The impression on C.T scan was that of Gastrointestinal stromal tumor arising from Sigmoid colon. 
Colonoscopy was done to locate the site of lesion, but was normal up to the caecum.

Patient was given 3 pints of blood preoperatively and then was decided to explore her abdomen. On laparotomy we found around $300-350 \mathrm{ml}$ of blood in the pelvis, $10 \times 10 \times 8 \mathrm{~cm}$ large encapsulated mass arising from the sigmoid mesocolon, freely mobile and separate from the sigmoid colon wall. There was no significant abdominal lymphadenopathy or tumor nodule.

The sigmoid colectomy with wide local excision of the tumor in the sigmoid mesocolon was performed with adequate proximal $(5 \mathrm{~cm})$ and distal margin $(2 \mathrm{~cm})$ and descending colon to rectum anastomosis was done .Post operatively patient was kept nil by mouth for 3 days till her bowel sounds returned and then started on full diet. The entire post operative course of the patient was uneventful and her symptoms resolved completely during the hospital stay.

On gross pathological study, the tumor was found to be soft, fleshy with areas of haemmorhage at few places. The mucosal surface of bowel was slightly congested but otherwise unremarkable. The sigmoid mesocolon didn't show any other nodule or lymph nodes.

On microscopic examination, the tumour was well within the resected margins. The colonic wall up to the serosa was essentially unremarkable. The tumor consisted of interlacing broad fascicles of plump spindly cells and moderately abundant superimposed lymphoplasmocytic infiltrate. Immunohistochemistry of the tumor were positive for CD45, CD68, and smooth muscle actin. The histopathological impression of the tumor was inflammatory myofibroblastic tumor of sigmoid mesocolon.

DISCUSSION: IMTs are challenging lesion with respect to classification, differential diagnosis and biological potential. Recently considered of having neoplastic nature, they are characterized by pseudosarcomatous proliferation of spindle cells in inflammatory fibrous stroma(3).Initially described in 1937, IMTs are synonymous for inflammatory solid tumor that contain spindle cells, myofibroblast, plasma cells and histiocytes ${ }^{(2)}$.

However there are some pathological aspects of IMTs that seemingly contradicted its purely inflammatory nature, including its potential for local recurrence, development of multifocal, non-contagious tumor, infiltrative local growth, vascular invasion and malignant transformation. In addition, the tumors have clonal characteristic, therefore IMTs should be probably regarded as soft tissue mesenchymal tumor with indeterminate or low malignant potential (13).

Females are often affected as commonly as males; the commonest site of IMT is lung which affect various age group ranging from 1-77 years with its peak at mid adulthood. This tumor is rare with an incidence of $0.04-26 \%$ for tumors in the lung. The incidence of this tumor in the colon is very rare. It has also been reported in variety of extrapulmonary sites, affecting younger patients(mean age 9.7 yrs ), with its peak at $1^{\text {st }}$ and $2^{\text {nd }}$ decade of life ${ }^{(4,6)}$.The extrapulmonary IMTs are rare, large in size, multinodular, less circumscribed and tend to be aggressive(3,8).

The presenting symptoms are nonspecific and depend on location. As a result differentiation from neoplasm cannot be based on clinical findings alone. In 15- $30 \%$ of cases , it may be accompanied by fever, growth failure, weight loss, hypochromic anaemia, thrombocytosis, polyclonal hyperglobulinaemia and raised ESR .Interleukin-6, Interleukin-1 and Cyclin-d production by the tumor are apparent cytokine mediators of this clinical and laboratory anomalies which may persist for months before IMTs are diagnosed and improves within days or few weeks after removal of tumor $(3,7,9,10)$. 
The microscopic pattern is polymorphic containing compact spindle cells arranged in haphazard fashion (with or without pleomorphism), myofibroblast, plasma cells and histiocytes (7,9) . On Immunohistochemistry, most spindle cells show strong diffuse reactivity with antibody against vimentin, muscle specific actin, but no or slight staining with desmin and cytokeratin(3).

The neoplastic nature of IMTs was demonstrated in 1991. Recently introduced anaplastic lymphoma kinase (ALK) -1 expression by most IMTs and not by other spindle cell tumors appears to be more specific marker. Recently Tsuzuki et al demonstrated that $75 \%$ of IMTs expresses ALK-1 which is confirmed by fluoroscene in situ hybridization and is highly $\operatorname{specific}^{(11)}$.

The tumor, mimics clinically and radiologically as malignant tumors especially sarcomas, however this tumor tends to be localized in majority of cases and even local recurrence is rare. Distant metastasis, which is again uncommon, is present at the time of presentation or within months or few years after the appearance of tumor (12).

On Computed tomography this lesion appears as attenuating soft tissue mass without any calcification. Mild enhancement is seen on post contrast study. There is usually no evidence of central necrosis. The reticulation of adjacent fat is an important finding and may suggest an inflammatory origin of the mass ${ }^{(1)}$.

Complete resection is the treatment of choice even in those with multiple recurrence . There is no proven role of chemo or radiotherapy in the management of IMTs $(5,12)$.

Long term follow up is highly recommended to detect local recurrence or possible metastasis which may develop many years later and to confirm benign nature of this tumor ${ }^{(1)}$.

\section{REFERENCES:}

1. Jain S, Bhargava SK,Upreti L,Mohta L. Inflammatory pseudotumor of sigmoid mesocolon. Indian journal of Radioimaging 2004:14:103-4.

2. Dehner LP, Kaye V, Levitt C. Cellular inflammatory pseudotumor in young individuals: a lesion distinguishable from fibrous histiocytoma or myosarcoma? Lab Invest. 1981; 44: $14 \mathrm{~A}$.

3. Coffin CM, Watterson J, Priest JR, Dehner LP. Extrapulmonary inflammatory myofibroblastic tumor (inflammatory pseudotumor): a clinicopathologic and immunohistochemical study of 84 cases. Am J Surg Pathol. 1995; 19: 859 - 872.

4. Souid AK, Ziemba MC, Dubansky A, Mazur M, Oliphant M, Thomas FD, et al. Inflammatory myofibroblastic tumor in children. Cancer. 1993; 72: 2042 - 2048.

5. Pettinato G, Manivel JC, De Rosa N, Dehner LP. Inflammatory myofibroblastic tumor (plasma cell granuloma). Clinicopathologic study of 20 cases with immunohistochemical and ultrastructural observations. Am J Clin Pathol. 1990; 94: 538 - 546.

6. Ro JY, Ayala AG, Ordonez NG, Swanson DA, Babaian RJ, et al. Pseudosarcomatous fibromyxoid tumor of the urinary bladder. Am J Clinic Pathol. 1986; 86: 583 - 590.

7. Shimer R, Helwig EB. Inflammatory fibroid polyps of the intestine. Am J Clinic Pathol. 1984; 81: $708-714$.

8. Tsuzuki T, Magi-Galluzzi C, Epstein JI. ALK-1 expression in inflammatory myofibroblastic tumor of the urinary bladder. Am J Surg Pathol. 2004; 28: 1609 - 1614.

9. Petridis AK, Hempelmann RG, Hugo HH, Eichmann T, Mehdorn HM. Metastatic low-grade inflammatory myofibroblastic tumor (IMT) in the central nervous system of a 29-yearold male patient. Clin Neuropath. 2004; 23: 158 - 166. 
10. Coffin.CM, Humphrey PA,DehnerLP:Extrapulmonary Inflammatory myofibroblastic tumor :clinical and pathological survey.Semin Diagnostic pathology 1998 May:15(2),85110.

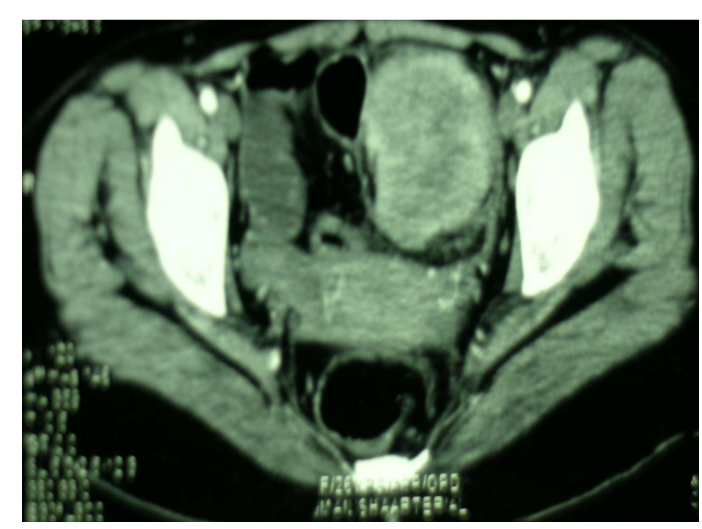

CT findings showing a tumor in the sigmoid mesocolon

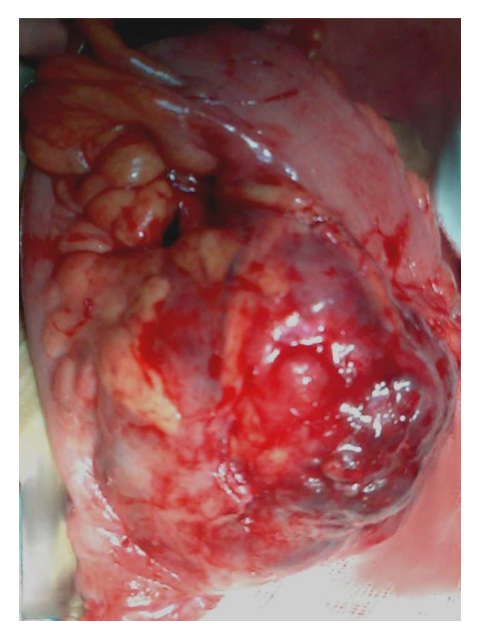

Photograph of the excised tumor. 